\title{
Effect of proline-enriched abalone feed on selected metabolite levels of slow-growing adult Haliotis midae
}

\author{
Leonie Venter $^{1}$ | Lodewyk Japie Mienie ${ }^{1}$ | Andre Vosloo ${ }^{2}$ | Du Toit Loots ${ }^{1}$ | Peet Jansen \\ van Rensburg ${ }^{1}$ | Jeremie Zander Lindeque ${ }^{1}$ (D)
}

${ }^{1}$ Human Metabolomics, North-West University, Potchefstroom, South Africa

${ }^{2}$ School of Life Sciences, University of KwaZulu-Natal, Durban, South Africa

Correspondence

Jeremie Zander Lindeque, Human

Metabolomics, North-West University,

Potchefstroom, South Africa.

Email: Zander.Lindeque@nwu.ac.za

\begin{abstract}
Abalone is currently considered South Africa's most successfully produced aquaculture export product, with a $76 \%$ share of the total value generated by the aquaculture sector. A major risk factor for this sector is slow growth rates experienced during farming. Abalone feeds are often supplemented with amino acids in an attempt to enhance abalone growth. This is a first investigation of the effect of added proline to standard abalone feed, on the metabolite profile of slow-growing abalone. A targeted liquid chromatography tandem mass spectrometry metabolomics research approach was followed to recognise the metabolic response of abalone showing slower growth performance. The addition of proline to the standard abalone diet was found to serve as a substrate for amino acid catabolism in slower growing abalone, by means of proline breakdown to assist with energy production via the tricarboxylic acid cycle. Other amino acids and urea cycle intermediates, that is, arginine, asparagine, ornithine and creatine further support energy production via the action of protein catabolism in slow-growing abalone. Additionally, the importance of understanding how abalone respond metabolically to modified feed highlights the use of metabolomics to answer abalone aquaculture farming questions.
\end{abstract}

KEYWORDS

aquaculture, Haliotis midae, liquid chromatography tandem mass spectrometry, metabolism, proline

\section{1 | INTRODUCTION}

In South Africa, the locally produced abalone, Haliotis midae, is an economically important aquaculture species, favoured internationally for its large size and organoleptic properties (Difford, Vlok, Rhode, \& Brink, 2017). Prior to animal export, slow and irregular growth rates are some of the major concerns. Research initiatives ranging from improved diet formulations, probiotic additives, system designs and water quality, to genetic improvement (Macey \& Coyne, 2005; Troell et al., 2006; Vlok, Difford, Rhode, \& Brink, 2016) have been implemented to improve animal growth and bridge the gap between fast and slow-growing animals.
Formulated feeds utilise fishmeal to ensure a high protein diet to provide the amino acids required for superior growth rates (Angell, Pirozzi, Nys, \& Paul, 2012; Green, Jones, \& Britz, 2011). The physiological importance of amino acids as components of protein building blocks and substrates for the synthesis of other molecules (Wu et al., 2014) are well described in literature. Supplementation of plantbased fish feed with lysine, methionine, threonine and tryptophan is considered a cost effective approach to supplement amino acids low in abundance (Li, Mai, Trushenski, \& Wu, 2009). It has also been shown in pigs and poultry, that a diet supplemented with lysine, tryptophan and methionine or cysteine, improve tissue protein synthesis and growth (Wu et al., 2014). Furthermore, a diet supplemented 
with L-proline was shown to significantly increase the growth rates in young mammals and chickens (Vosloo, Rensburg, \& Vosloo, 2013).

The importance of proline has been widely demonstrated to play an important role in cell structure, wound healing, anti-oxidative reactions, immune responses, energy metabolism and protein synthesis in numerous organisms (Wu et al., 2011). In addition, Wu et al. (2011) promotes proline as the most important amino acid supporting whole body protein synthesis. Proline was also found to protect proteins from damage, due to its ability to act as a free radical scavenger and subsequently, protect the peptide backbone of proteins (Vosloo et al., 2013). In plants, proline accumulation occurs in response to osmotic stress, temperature variations, nutritional deficits, bacterial pathogens and heavy metals (Pérez-Arellano, Carmona-Álvarez, Martínez, Rodríguez-Díaz, \& Cervera, 2010). This study, however, focuses on the mechanism by which proline may enhance mitochondrial activity and support energy production (as displayed in Figure 1).

When proline is oxidised to pyrroline-5-carboxylate (P5C), proline dehydrogenase transfers reducing equivalents to the electron transport chain (ETC) (Krishnan, Dickman, \& Becker, 2008). Together with the subsequent activation of electron transfer flavoprotein (ETF), P5C has been found to be beneficial to the maintenance of osmotic and redox balance (Pérez-Arellano et al., 2010). While the conversion of proline to P5C supplies electrons to the ETC (Teulier,
Weber, Crevier, \& Darveau, 2016) and supports energy production, the reactions of ETF (together with increased proline) appear to have an effect on cell volume, affecting the osmotic strength of cells, accompanied by an increase in cytoplasmic calcium $\left(\mathrm{Ca}^{2+}\right)$ (Halestrap, 1987). Abundant proline, and its mitochondrial oxidation, has been demonstrated to elevate the cell's osmotic capacity, the subsequent increase in cell matrix volume, and the resulting osmotic swelling of the cell (Ballantyne \& Moyes, 1987). With an increased matrix volume, increased rates of beta( $(\beta)$-oxidation would be expected, as was previously demonstrated in liver cells stimulated by $\mathrm{Ca}^{2+}$ (Halestrap, 1987). Altogether it seems probable that an increase in proline intake would increase ( $\beta$ )-oxidation, with the resulting increase in acetylcoenzyme A ( COA) being utilised by the tricarboxylic acid (TCA) cycle intermediates and energy production processes. In effect, increased energy production will stimulate the release of insulin which plays a key role in glucose and amino acid uptake and the synthesis of protein, fat and glycogen (Newsholme, Bender, Kiely, \& Brennan, 2007).

In a short-term study on the benefits of proline-supplemented abalone feed, Vosloo et al. (2013) proposed that the enhanced antioxidant capabilities provided by proline supplementation might contribute to production level growth benefits when extended over longer-term studies. Coupled to recent work on fast-growing $\mathrm{H}$. midae consuming standard abalone feed, Abfeed ${ }^{\mathrm{TM}}$ (Marifeed Pty Ltd, South Africa), where it was hypothesised that faster growth can be

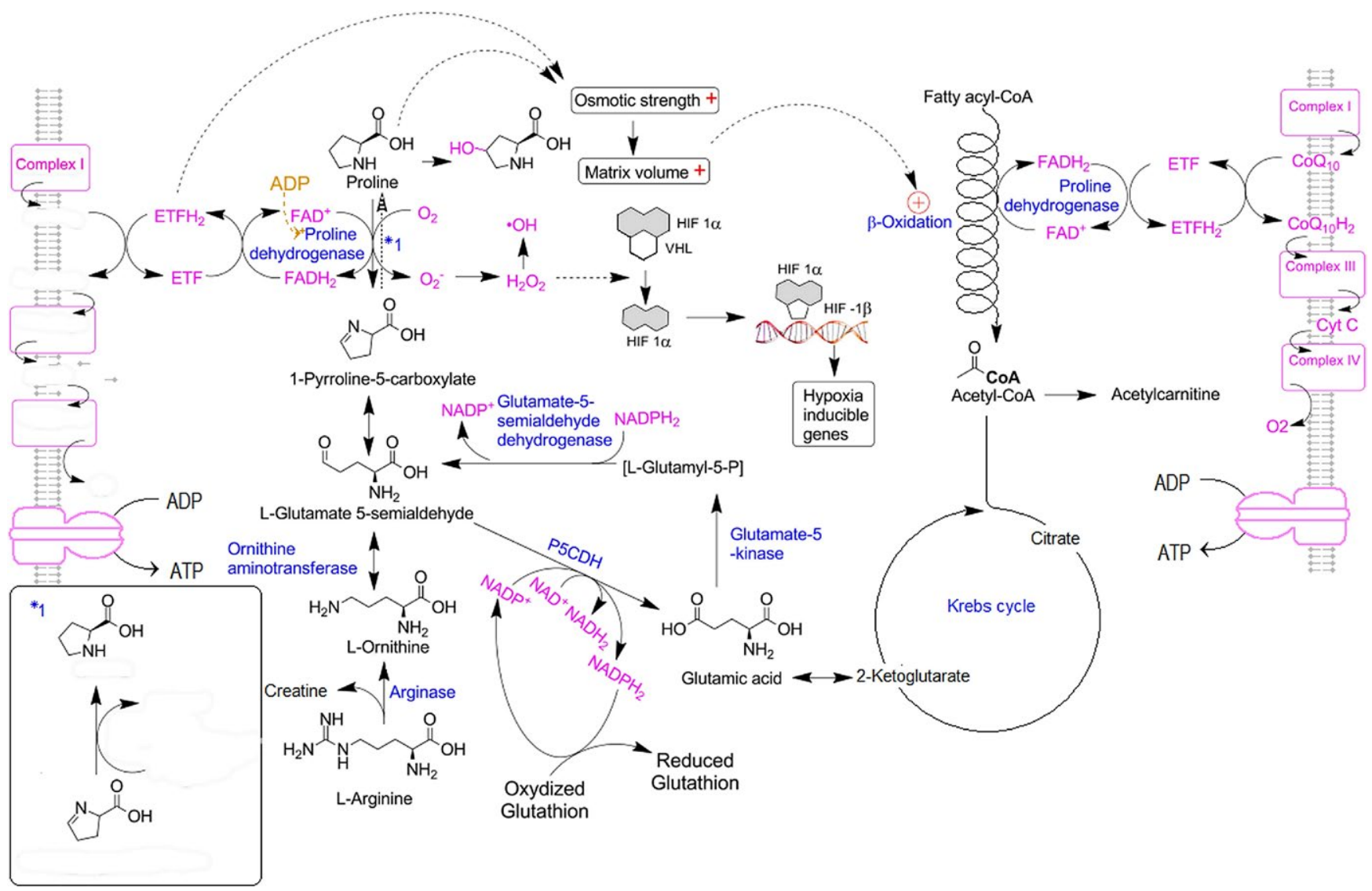

FIGURE 1 Mechanistic insights into proline metabolism. Proline enhances mitochondrial activity by stimulating cellular osmotic stress, which supports increased fatty acid catabolism resulting in energy production, and proline activates hypoxia inducible genes that play a protective role during hypoxia 
linked to elevated insulin production which promotes protein synthesis (Venter, Vosloo, Mienie, Rensburg, \& Lindeque, ), it becomes clear that investigations into the metabolic underpinnings of enhanced growth in farmed abalone are of major commercial importance. Even more so when focusing on the part of the abalone which is considered the sought after edible part, commonly canned or frozen for exportation. Abalone are grown and cared for with the sole purpose of supplying abalone (more specifically muscle) of market size to the demanding market. The foot region constitutes about $66 \%$ of the total body mass of abalone, and is comprised of different muscle fibre types, which are expected to have different metabolic end products (Venter, Loots, Vosloo, Jansen Van Rensburg, \& Lindeque, 2018).

The previously unanticipated importance of amino acid and fatty acid (acylcarnitines) dependent pathways in abalone-fed formulated feeds was recently demonstrated using targeted metabolomics analysis by means of liquid chromatography tandem mass spectrometry (LC-MS/MS), providing robust support for the use of the analytical platform (Venter et al., 2018b). The use of LC-MS/MS in multiple reaction monitoring (MRM) mode helps to analyse target compounds based on the transition from the unique precursor ion in the first quadrupole and selected product ion in the second quadrupole mass analyser, resulting in a higher specificity and selectivity when analysing compounds in this manner (George et al., 2010); and have proved useful in nutrition-related studies (Xu et al., 2018). Considering the specificity offered by LC-MS/MS and the groundwork that has already been covered, the aim of this study was to investigate the effect of proline-enriched abalone feed on the metabolite profile of slow-growing adult $\mathrm{H}$. midae, using a targeted metabolomics approach.

\section{2 | MATERIALS AND METHODS}

The procedures and protocols used for this research were ethically reviewed and approved in accordance with the guidelines of the relevant institutional committees and granted Aquaculture Research Permit.

\section{1 | Experimental design at production level}

An 8-month abalone growth experiment was conducted on-site at HIK Abalone Farm (Pty) Ltd, situated in Hermanus, South Africa. The same cohort of animals was monitored for the duration of the experiment allowing day-to-day farming activities and regular size grading to continue as per farm protocol. The animals were subjected to two different feeding regimes. The first feeding regime used the standard formulated abalone feed (Abfeed S34) (Marifeed (Pty) Ltd, Hermanus, South Africa), and the second used proline-supplemented Abfeed (Vosloo et al., 2013), which had an identical formulation to Abfeed S34, but was supplemented with $10 \mathrm{~g}$ proline per kilogram dry feed.

Eight raceways, each containing 12 baskets of abalone (approximately 27 months of age; $65 \mathrm{~g}$ wet weight; $71 \mathrm{~mm}$ shell length), were used for the growth trial. Following 8 months of abalone farming, 48 baskets of adult abalone (with each basket containing approximately 190 animals) consuming standard abalone feed and 48 baskets of abalone proline-enriched abalone feed were subjected to grading (weighing animals individually) and splitting (redistribution of biomass to maintain stocking density) based on the standard protocol of the farm. These data allowed for the calculation of farm-relevant growth data per basket per month for both feeding regimes.

\section{2 | Experimental design for metabolomics analysis}

Within the growth experiment, (described in Section 2.1) sampling of abalone adductor muscle was conducted at the onset of the experiment and again after 8 months of farming from the two cohorts of abalone consuming different feeds. At time of sampling, animals were further divided into two growth classes, for example, slow growers and fast growers, based on their weight and length. Abalone were defined as a slow grower if the animal was visibly smaller than other abalone in the same sampling basket at an identical life stage. Conversely, abalone was defined as a fast grower if a larger size was visibly seen in an individual amongst animals from the same sample basket at the same life stage. Prior to dissection, the animals were weighed to the nearest $0.01 \mathrm{~g}$ and the shell lengths were measured to the nearest $0.1 \mathrm{~mm}$, along the longest axis, using callipers. The weight and length data further confirmed the size differential based on visual observations. Abalone gender was assessed before dissection based on the colour of the gonad, which is a cream colour in males and a green colour in females.

At time of sampling, 36 abalone (18 abalone consuming standard abalone feed and 18 abalone consuming proline-enriched abalone feed) were removed from their respective holding systems, and gently patted dry with paper towels. Sampling measures for abalone consuming standard abalone feed consisted of: nine slow-growing abalone ( 4 male and 5 female) with an average wet weight of $85.70 \pm 8.47 \mathrm{~g}$ and shell length of $79.80 \pm 1.56 \mathrm{~mm}$, and nine fastgrowing abalone ( 5 male and 4 female) with an average wet weight of $124.00 \pm 11.50 \mathrm{~g}$ and shell length of $87.90 \pm 3.38 \mathrm{~mm}$. The abalone consuming proline-enriched abalone feed group contained: nine slow-growing abalone ( 5 male and 4 female) with an average wet weight of $90.40 \pm 9.89 \mathrm{~g}$ and shell length of $79.70 \pm 2.11 \mathrm{~mm}$, and nine fast-growing abalone (6 male and 3 female) with an average wet weight of $121.30 \pm 10.62 \mathrm{~g}$ and shell length of $88.10 \pm 2.36 \mathrm{~mm}$.

\subsection{1 | Tissue sample collection}

Animals were shucked using a scalpel, working rapidly from the anterior to posterior axis, cutting longitudinally through the foot between the mantle and the distal surface of the adductor muscle. A sample of the shell adductor muscle was removed from the 36 abalone sacrificed for metabolomics analysis, using a scalpel from the ventral surface towards the central point where the muscle attaches to the shell. Muscle samples to the equivalent of the volume of a $2 \mathrm{ml}$ micro-centrifuge tube were sampled in small blocks using a scalpel 
and snap frozen using dry ice, before shipment, on dry ice, to the North-West University.

\subsection{2 | Metabolite extraction and sample preparation}

A subsample $(50 \mathrm{mg})$ of muscle tissue was removed with a scalpel (while on ice), transferred into a new tube and weighed for the purpose of pre-analysis normalisation. Tissue homogenisation was performed using a two-step method previously published (Venter, Jansen Van Rensburg, Loots, Vosloo, \& Lindeque, 2016). In brief, $50 \mathrm{mg}$ of the adductor muscle tissue was placed into separate microcentrifuge tubes, followed by the addition of $200 \mu$ l water, $560 \mu$ l methanol and $60 \mu \mathrm{l}$ internal standard (2-acetamidophenol and 3 phenylbutyric acid, with a final concentration of $50 \mu \mathrm{g} / \mathrm{ml}$ ), along with both a 3- and 7-mm stainless steel bead. This was homogenised for 4 min at $30 \mathrm{~Hz}$ using a Retch M400 vibration mill. Subsequently, $180 \mu \mathrm{l}$ chloroform and $90 \mu \mathrm{l}$ water was added and each tube was vortexed for $1 \mathrm{~min}$, and then incubated on ice for $10 \mathrm{~min}$. The samples were centrifuged at $25,000 \mathrm{~g}$ for $10 \mathrm{~min}$ at $4^{\circ} \mathrm{C}$ resulting in phase separation. A volume of $60 \mu \mathrm{l}$ upper polar phase and $30 \mu \mathrm{l}$ of the lower a-polar phase were used for LC-MS/MS analysis, and an isotope mixture was added to ensure the monitoring of a known compound for retention time comparisons between batches. This combined sample was dried under a gentle stream of nitrogen at $37^{\circ} \mathrm{C}$. To the dried residue, $100 \mu \mathrm{l} 3 \mathrm{~N}$ butanolic $\mathrm{HCl}$ solution (prepared 1:4 acetyl chloride:1-butanol ratio) was added, whereafter the samples were capped and incubated at $60^{\circ} \mathrm{C}$ for $60 \mathrm{~min}$. The butylated samples were evaporated to dryness under a gentle stream of nitrogen at $60^{\circ} \mathrm{C}$. Lastly, the dried residue was reconstituted in $200 \mu \mathrm{l}$ water:acetonitrile (50:50) containing $0.1 \%$ formic acid (Venter, Jansen Van Rensburg, Loots, Vosloo, \& Lindeque, 2017).

\subsection{3 | Sequence and batch design}

Samples were assigned to a batch using a randomisation equation to allocate these to the respective batches for analysis (Dunn, Wilson, Nicholls, \& Broadhurst, 2012). Quality control (QC) samples were included in every batch by preparing a pooled mixture of the adductor muscle tissue. These QC samples were included within the biological sample batches and treated identically to the experimental samples. The QC samples were injected at regular intervals throughout the analytical run of the analysed batch.

\subsection{4 | LC-MS/MS conditions}

Reverse phase liquid chromatography analyses were performed using an Agilent 1200 Infinity LC system as previously described (Venter et al., 2018a). In brief, a sample volume of $0.5 \mu \mathrm{l}$ was injected onto an Agilent SB-Aq column $(2.1 \times 100 \mathrm{~mm}, 1.8 \mu \mathrm{m})$ maintained at $45^{\circ} \mathrm{C}$. Water $(\mathrm{A})$ and acetonitrile (B) both with $0.1 \%$ formic acid were used as mobile phases for separation under the following gradient conditions: 0 min 5\% (B); 0-0.2 min 5\% (B); 0.2-2 min 25\% (B);
2-7 $\min 25 \%(\mathrm{~B}) ; 7-7.5 \min 90 \%(\mathrm{~B}) ; 7.5-9 \min 90 \%$ (B) all using a flowrate of $0.4 \mathrm{ml} / \mathrm{min}$; 9-9.1 $\mathrm{min} 90 \%$ (B); 9.1-12 $\min 95 \%$ (B) and 12-12.5 min 5\% (B). The remaining gradient was executed using a flowrate of $0.5 \mathrm{ml} / \mathrm{min}$ followed by a $4 \mathrm{~min}$ post-run (total run time of 16.5 min per sample). Mass spectrometry detection was performed on an Agilent 6410 Triple Quadrupole using positive electrospray ionisation, a drying gas temperature of $300^{\circ} \mathrm{C}$, a drying gas flow of $7.5 \mathrm{~L} / \mathrm{min}$ and nebuliser pressure of $30 \mathrm{psi}$. MRM transitions were optimised via direct infusion with Agilent Technologies MassHunter optimizer software (Venter et al., 2018a). All source conditions were kept at the predetermined optimum values for each metabolite with a dwell time of $45 \mathrm{~ms}$. Table 1 summarises the optimised source and MRM conditions for each metabolite using enhanced sensitivity at an EMV of 400 displaying the monitored metabolites, the precursor and product ions [mass to charge ratio $(\mathrm{m} / \mathrm{z})$ ] selected, the fragmentor $(F)$ voltage and the collision energy (CE) voltage used.

\subsubsection{LC-MS/MS data analysis}

Data were inspected with Agilent's MassHunter Qualitative software for retention time drifts and other batch-related effects before quantification. Supervised peak integration was performed using Agilent's MassHunter Quantitative software.

\subsection{6 | Data processing}

\section{Data quality evaluation}

The quality of the data was firstly evaluated to ensure that technical variance from experimental factors did not affect the relevant biological variance and that the data could be used without corrections. The QC samples analysed amongst the batch were assessed in terms of coefficient of variance percentages and visual interpretations (Wehrens et al., 2016). The data were evaluated for a within batch effect (Xia, Mandal, Sinelnikov, Broadhurst, \& Wishart, 2012), and as no visible effect was seen within the datasets no correction methods were applied. Furthermore, principal component analysis (PCA) was used to determine whether a natural grouping in the data exists when considering this from a multivariate perspective (Lindeque et al., 2015). Factors like gender, size and time of sample preparation were investigated using this approach in order to confirm that the relevant biological variance in the compared groups was not influenced by these confounders. As a result, the grouping displayed visually on the PCAs did not display any favour towards possible confounding factors within the groups of interest.

\section{Data pre-processing}

Zero filtering was performed (Venter et al., 2015), by removing features not detected in all of the samples of one experimental group, as they do not contain any statistical value.

\section{Data normalisation}

To remove non-biological variation, the data were normalised using the mass spectrometry total useful signal normalisation method. 


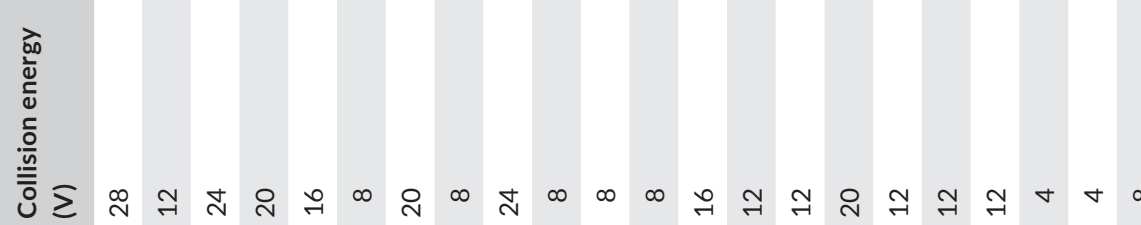

徆

으

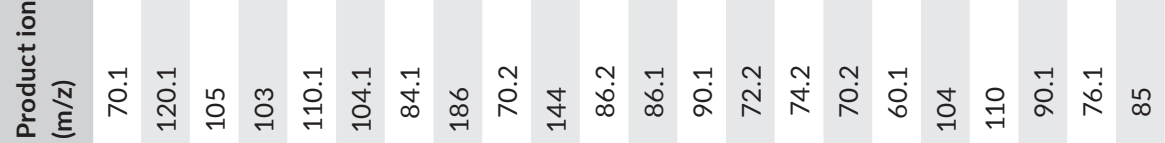

$\frac{\overline{0}}{\frac{\bar{t}}{2}}$

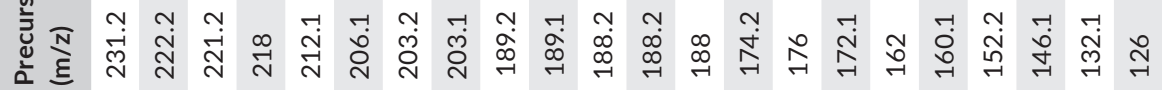

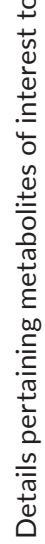

$\stackrel{\dot{y}}{\frac{\dot{ \pm}}{0}}$

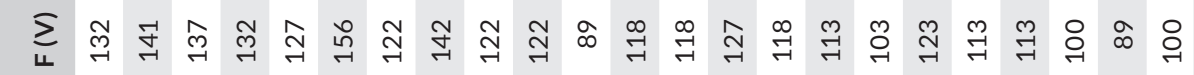

흔

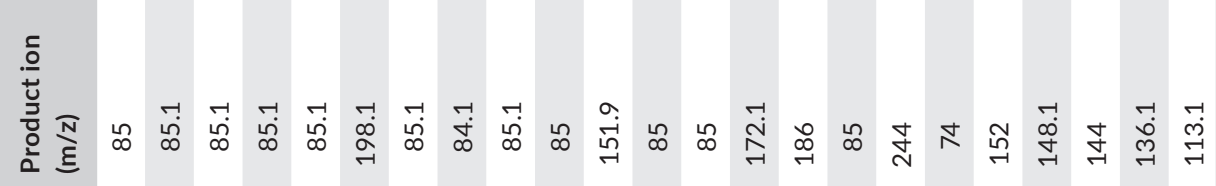

$\frac{.}{\frac{\bar{t}}{\circ}}$

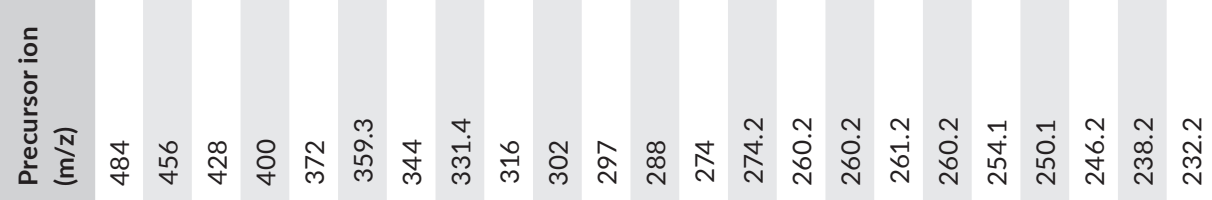

ن

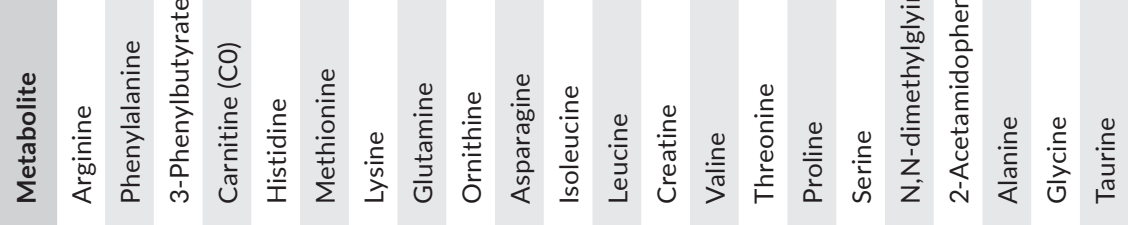


wwThis method performs correction in accordance with the sum of all the component signals that are present in all the samples (Lindeque, Matthyser, Mason, Louw, \& Taute, 2018).

\section{Data pre-treatment}

The pre-treatment steps were completed by using the webserver MetaboAnalyst (www.metaboanalyst.ca) (Xia, Sinelnikov, Han, \& Wishart, 2015). Firstly, the missing value estimation was performed by replacing the missing (or zero) values with half of the minimum positive value in the original data. The data were further transformed using the generalised logarithm (glog) transformation function before statistical analysis. This ensures a more normal distribution of the metabolite values for parametric tests. Also, it decreases the weight of the more abundant compounds for multivariate analysis (Lindeque, Hidalgo, Louw, \& Westhuizen, 2013).

\section{Statistical analysis}

The average mass increase per basket per month was compared using an unpaired $t$ test with Welch's correction for unequal variances $(p<0.05)$ after normality testing (Shapiro-Wilk Test, $p>0.05$ ).

For metabolomics data, multivariate analyses were done to get an overview of the data and to observe the covariance of metabolites and differences in metabolite levels between individuals consuming both standard and proline-enriched abalone feed (Lindeque et al., 2015). Univariate statistical analyses were used to identify biological meaningful variables, for the purpose of identifying the metabolite markers best describing the variation between the slow and fast-growing abalone consuming proline-enriched abalone feed.

MetaboAnalyst was used to perform PCA analysis on the entire dataset to visualise the differences between the experimental groups. Scatter plots of the PCA scores were plotted with $95 \%$ confidence ellipses to more effectively indicate grouping. Univariate analyses were performed with MetaboAnalyst and MS Excel. Student's $t$ test was used to find significant differences in metabolite levels between the experimental groups of the proline-enriched feed group. Features with a $p<0.05$ (false discovery rates corrected $p$-value) indicated that the features differed significantly between groups and were labelled as important. The features with a $p>0.05$ were removed from the data matrix, resulting in only data with statistical significance. The effect size of the remaining features was determined to ensure that the differences were large enough (or practical significant). Effect size was calculated by determining the absolute difference between the means of the two groups divided by the maximum standard deviation of the two groups. Features with a $d>0.8$ were labelled as important and remained in the data matrix for further analysis (Ellis \& Steyn, 2003). Using the calculated $p$-values and $d$-values, a Volcano plot was constructed to visualise the statistical results differentiation between slow and fast-growing abalone.

\section{Pathway analysis}

The important metabolites that differed statistically and practically between the slow and fast-growing experimental group consuming enriched abalone feed were used for pathway analysis. A metabolic map of the metabolite profile of slow-growing adult $H$. midae was compiled manually. Pathways where several metabolites differed significantly between the experimental groups were considered to be affected. The metabolites are reported as relative compound intensities based on their presence in the abalone adductor muscle sample.

\section{3 | RESULTS}

\section{1 | Production level farm data}

A mean mass difference of $122 \pm 24.5 \mathrm{~g} /$ basket/month for adult abalone consuming proline-enriched abalone feed was calculated (Figure 2) when compared to the mass increase of abalone consuming standard abalone feed.

\section{2 | Multivariate statistical analysis of abalone consuming both standard and proline-enriched abalone feed}

A total of 29 metabolite detected were used to construct the PCA score plot (Figure 3 ) depicting abalone which consumed standard and proline-enriched abalone feed with further distinction been slow and fast-growing abalone groups. The first two principal components accounted for $31 \%$ of the total variance in the data. The PCA score plot shows differentiation between the slow $(X)$ and fast $(\Delta)$ growing abalone consuming standard abalone feed and an overlap between slow $(+)$ and fast ( 0 ) growing abalone consuming proline-enriched abalone feed, with no other outliers or subgroupings present.

\section{3 | Univariate statistical analysis of slow and fast- growing abalone consuming proline-enriched abalone feed data}

The muscle metabolites that differed markedly between the slow and fast-growing abalone consuming proline-enriched abalone

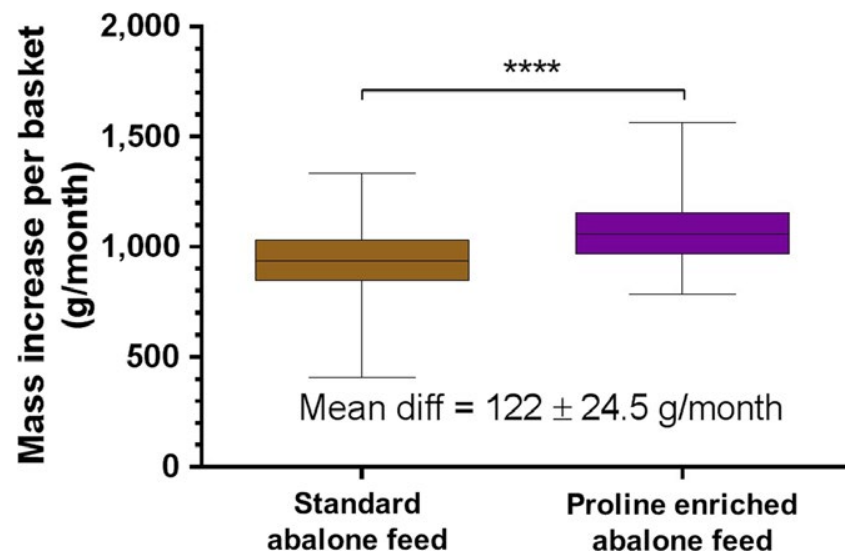

FIGURE 2 Farm data collected from 48 baskets of abalone subjected to an 8-month feeding trial consuming standard abalone feed (left) and proline-enriched abalone feed (right). The horizontal line, boxes and whiskers represent the median, 25-75th percentiles and range respectively. ${ }^{* * *}$ Indicate significance $(p<0.001)$. 


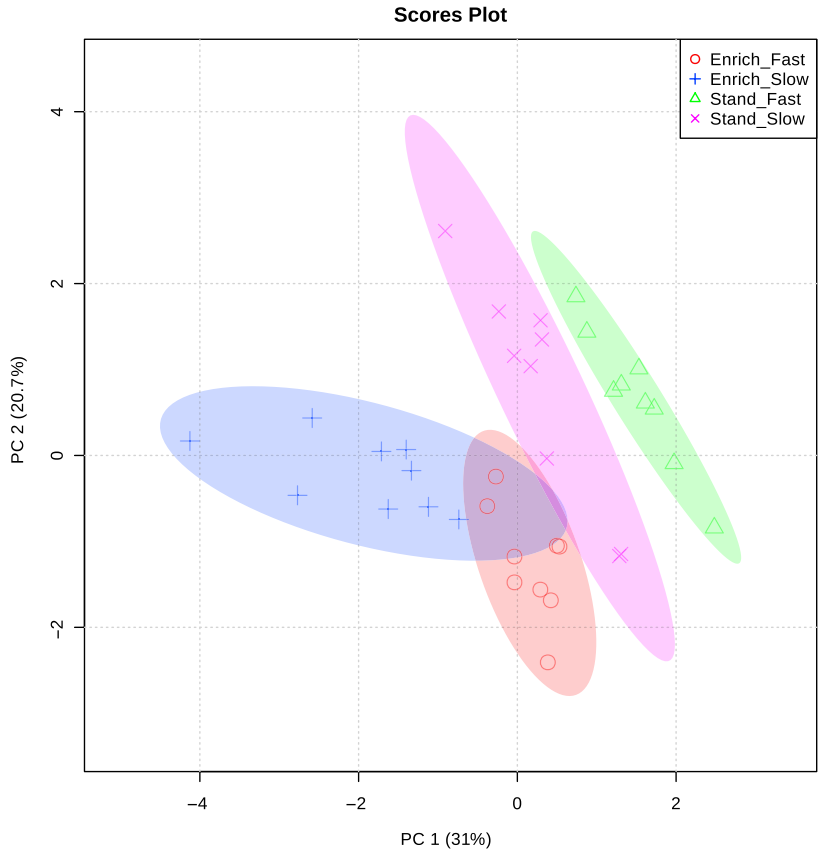

FIGURE 3 PCA score plot of adductor muscle metabolites in abalone consuming standard (stand) and proline-enriched abalone feed (enrich) with 95\% confidence ellipses showing grouping of fast and slow-growing adult abalone in both groups

feed are given in the Volcano plot of Figure 4, with effect size $\left(d^{2}\right)$ on the $x$-axis and $-\log 10$ adjusted $p$-value on the $y$-axis. Based on univariate analyses, a total of 10 metabolites showed an increased metabolic response, while eight metabolites displayed a reduced metabolic response.

\subsection{Metabolic differences between slow and fast-growing abalone consuming proline-enriched abalone feed}

Analysis of abalone muscle samples revealed that the metabolite profile (Figure 5) of slow-growing animals consuming proline-enriched feed. Here, acetylcarnitine and carnitine belong to the fatty acid metabolite class, while alanopine, creatine and tauropine are classified as metabolites of carbohydrate metabolism. Lastly, the amino acid metabolite class containing alanine, arginine, asparagine, glycine, histidine, leucine, lysine, N,N-dimethylglycine, ornithine, proline, threonine tryptophan and tyrosine was most affected (Table S1).

\section{4 | DISCUSSION}

\section{1 | Improved growth rates with proline supplementation based on production level farm data}

The farm data collected from 48 baskets of abalone consuming proline-enriched abalone feed (Figure 2) suggests that this diet offers production level benefit when directly compared to standard abalone feed data. Based on research done on juvenile $\mathrm{H}$. midae, it was estimated that feed supplementation with L-proline provides individual advantage, potentially benefiting the mariculture industry (Vosloo et al., 2013). This outcome was seen once again when using adult animals and highlights the importance of understanding the metabolic changes induced by proline-enriched abalone feed as it may assist with increased abalone growth especially when investigating methods to assist with bridging of the gap between slow and fast-growing abalone (as discussed next).

\subsection{Overview of muscle metabolite data generated using metabolomics analysis}

A number of studies have recently proved the usefulness of metabolomics in discerning the effect of diet intervention (Jarak et al., 2018; Xu et al., 2018; Yang et al., 2018). As metabolomics is the endpoint of the 'omics cascade' and is the closest to the functional phenotype of the cell (Dettmer, Aronov, \& Hammock, 2007), the metabolome was investigated for any further phenotypic changes which may be induced in abalone consuming proline-enriched feed. The PCA score plot in Figure 3 gives an overview of the data prior to univariate analysis. Here, the metabolites detected in abalone adductor muscle analysed with LC-MS/MS analyses are shown with differentiation between slow and fast-growing abalone receiving standard and proline-enriched abalone feed.

The slow-growing abalone groups consuming standard $(X)$ and enriched $(+)$ abalone feeds show the largest variation which can be attributed to the large phenotypic (weight and length) variance found within these groups. When focusing on the fast-growing abalone group ( 0 ) consuming the enriched diet, the samples are grouped closer together demonstrating comparable metabolite profiles in this group of animals. The fast-growing group consuming standard abalone feed $(\Delta)$ grouped separately from the other groups, highlighting unique metabolic changes occurring in that group. Abalone grouping in accordance to size/age/growth classes is a common practice as demonstrated in $\mathrm{H}$. tuberculate where improved production was eventually achieved when same-size counterparts are grouped (Mgaya \& Mercer, 1994). The metabolomics data demonstrated here further shows covariance of the metabolites liked to specific groups, which can be used to discriminate between slow and fast-growing animals, serving as a useful for metabolite-guided classification in future studies. Lastly, the overlap seen in the confidence ellipses of the enriched groups suggests that there are similar metabolic processes at similar rates between the fast and slow-growing receiving added proline. In order to assess the underlying metabolic differences, the focus will now shift to solely the proline-enriched abalone group.

\subsection{Metabolic differentiation between slow and fast-growing abalone consuming proline-enriched abalone feed}

The Volcano plot differentiating between slow and fast-growing abalone consuming proline-enriched feed (Figure 4) clearly shows 


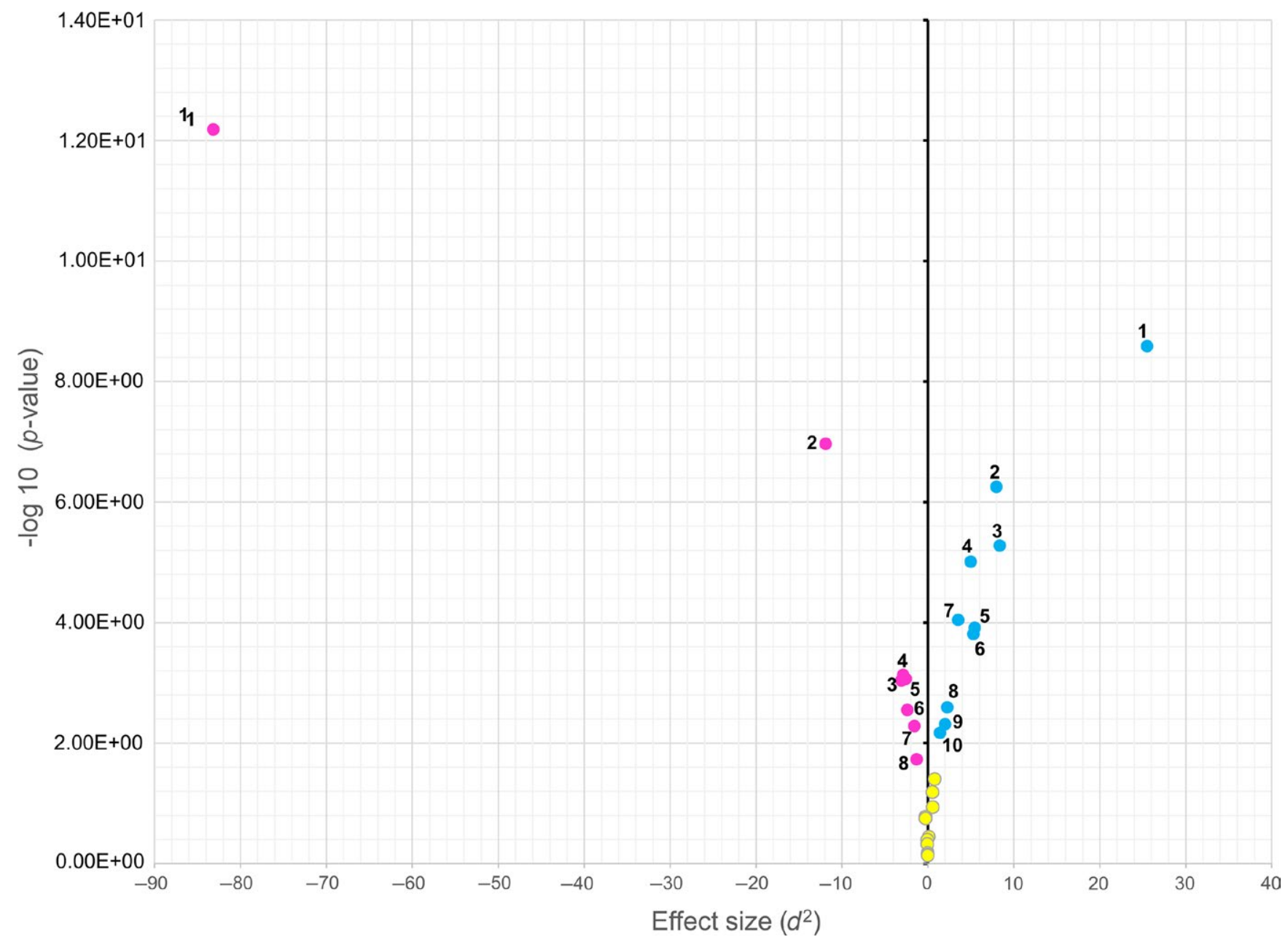

FIGURE 4 Volcano plot showing the metabolite differences between slow and fast-growing abalone consuming proline-enriched abalone feed. Increased metabolites (blue) include: (1) ornithine, (2) tryptophan, (3) carnitine, (4) arginine, (5) glycine, (6) creatine, (7) asparagine, (8) leucine, (9) tyrosine and (10) alanine. Decreased metabolites (pink) include: (1) histidine, (2) acetylcarnitine, (3) proline, (4) N,Ndimethylglycine, (5) tauropine, (6) alanopine, (7) threonine and (8) lysine

histidine as the metabolite which was the largest affected. Histidine is regarded as an essential amino acid in abalone and should be supplied by diet (Fleming, Barneveld, \& Hone, 1996), suggesting that histidine is not sufficiently present in abalone muscle following consumption of proline-enriched abalone feed. Numerous metabolomics studies have detected histidine in abalone (Bae, Yoon, \& Lim, 2011; Lu, Feng, Cai, \& Chen, 2017; Sheedy, Lachambre, Gardner, \& Day, 2015; Venter et al., 2018b; Viana et al., 2007), but none to date link this metabolite to growth differences of abalone, making it an interesting metabolite for future investigations.

When focusing on the effect of added proline to the standard abalone feed, it is important to keep in mind that both slow and fastgrowing abalone received added proline from a dietary source for metabolic use. However, the slow-growing group showed decreased proline levels suggesting catabolism (breakdown) thereof for usage in energy production (Figure 5). It appears as if the proline supplementation reversed the metabolic profiles of the slow-growing abalone when compared to their fast-growing counterparts as previously seen (Venter, Vosloo, Loots et al., 2018). Based on the previous work on $H$. midae, it was concluded that slow-growing abalone did not fully utilise glycolysis for energy production but also depends on alternative pathways (such as $\beta$-oxidation and amino acid catabolism) to sustain basal energy levels. Likewise, this study also demonstrates that slowgrowing abalone depend on alternative routes for energy production utilising predominantly amino acids like proline as an energy source.

From Figure 5, it is clear that proline ensures glutamate production to replenish alpha( $\alpha$ )-ketoglutarate levels in the TCA cycle following deamination (Brosnan, 2000), which is typically what is expected in the slow-growing abalone group. As part of regulatory functioning, the TCA cycle allows oxaloacetate to be converted to aspartate (and vice versa) (Newsholme et al., 2003). With additional proline catabolised, glutamine production is initiated ensuring glutamate production and conversion to the TCA cycle subsequently resulting in increased aspartate and then asparagine production. Furthermore the hydrolysis of asparagine yields aspartate (Garrett \& Grisham, 2010) which is required to produce argininosuccinate, and with that the production of arginine is stimulated, which cleaves to urea and ornithine, and also causes creatine production (Salway, 


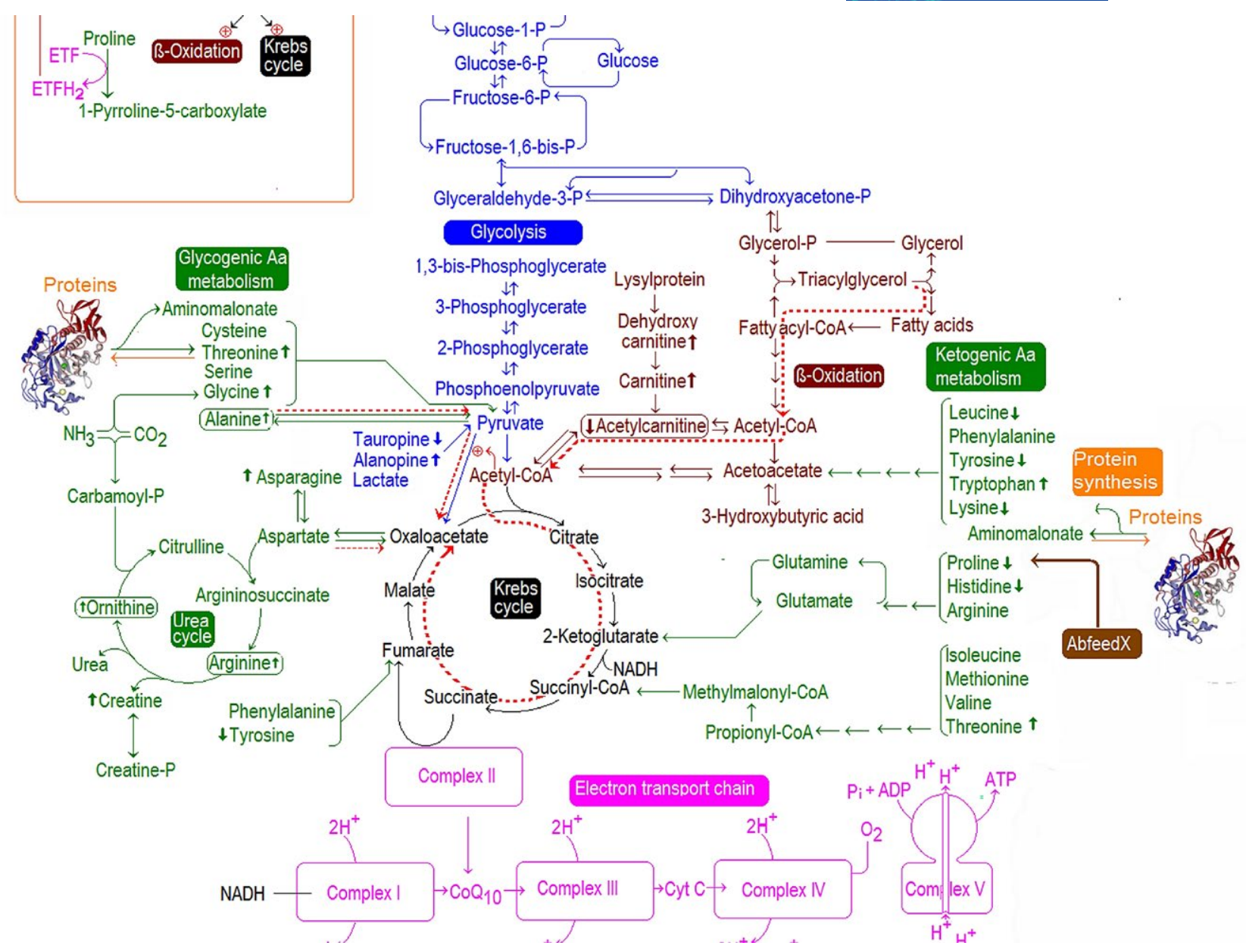

FIGURE 5 Metabolic muscle profile of slow-growing Haliotis midae with comparative concentrations of those metabolites significantly different in the carbohydrate (blue), amino acid (green) and fatty acid (brown) metabolic pathways, are indicated by an increase ( $\uparrow$ ) or decrease $(\downarrow)$ in comparison to that fast-growing abalone group

2004), of which increased metabolite concentrations were observed. The catabolism of proline releases an amino group which supports the production of ammonia resulting in urea production and increased urea cycle intermediates. Increased urea cycle intermediates are typically seen in abalone when subjected to hypoxic conditions; (Venter et al., 2018b), however, their presence may be linked to increased protein catabolism as a consequence of increased proline breakdown and downstream pathways. Furthermore, instead of phosphoarginine and phosphocreatine being broken down as an adenosine triphosphate (ATP) source, it seems probable that the increased intermediates will be converted to these phosphate sources to fill intracellular stores for future use.

The use of amino acids to stimulate protein synthesis is also demonstrated when viewing the amino acids with decreased concentrations in Figure 5. Decreased levels of essential amino acids like tyrosine, leucine, lysine and histidine support the usage for the synthesis of proteins, adding to the large metabolite variation seen in the slow-growing proline-enriched abalone group (+) of the PCA in Figure 3. Altogether, the process of protein synthesis is dependent on factors like substrate availability, the developmental stage of the organism, environmental factors, species etc. (Wu et al., 2014), making it impossible at this stage to speculate which amino acids are used primarily for protein synthesis, but considering the decrease of the above-mentioned metabolites, they can be considered as building blocks for protein synthesis.

Additional proline in the mitochondria also supports $\beta$-oxidation, which resulted in decreased acetylcarnitine and increased dehydroxycarnitine and carnitine in the slow-growing abalone group. In brief, $\beta$-oxidation is responsible for the breakdown of fatty acid molecules to generate acetyl-CoA for usage in the TCA cycle (Garrett \& Grisham, 2010). Then again acetylcarnitine can be regarded as storage product for acetyl-CoA production which also assists with TCA functioning and ATP production via the ETC in abalone during standard farming conditions. The activation of fatty acid catabolism in this study is also supported by the working of proline, which stimulates mitochondrial activity. Although no literature supporting this in abalone could be found, evidence for this has previously been reported in other species like bumblebees (Teulier et al., 2016). Research done on plants show that during stress, proline and lipid metabolism share dual roles (Shinde, Villamor, Lin, Sharma, \& Verslues, 2016), suggesting that proline has the ability to regulate $\beta$-oxidation, subsequently resulting in decreased acetylcarnitine and an increase in alanine, as seen in this 
experiment (Figure 5). Furthermore, adult abalone have the capacity to accumulate lipid stores, suggesting an increased dependence on a pathway like $\beta$-oxidation for energy production in $\mathrm{H}$. midae (Laas \& Vosloo, 2010). Based on a dietary lipid investigation conducted on $H$. iris, it was concluded that lipids do not significantly affect the growth of this abalone species (Tung \& Alfaro, 2012), which could be true for H. midae as well.

Although both groups consumed a proline-enriched diet, it appears that the slow-growing abalone were able to metabolise more of the amino acid compared to the fast growers. This might then also hint that amino acid catabolism is very active in the slow-growing adult abalone, which could explain their growth rate (as demonstrated by weight and length measures in Section 2.2).

\section{CONCLUSIONS}

Abalone feed supplementation with proline offers production level benefit and although the understandings of the metabolic underpinnings driving this are still controversial it is important for future directed feed improvements. Also, the findings of this study highlight the metabolic significance of added proline to abalone feeds. The addition of proline to standard abalone feed serves as a sufficient substrate for amino acid catabolism. The metabolite profile of slowgrowing abalone suggests that the breakdown of additional proline from a dietary source assists with energy production via the use of the TCA cycle and the ETC. This is further supported by increases in urea cycle intermediates and several amino acids which support the action of protein catabolism for energy production purposes. Lastly, this study is helpful for a better understanding of the metabolic functioning of slow-growing adult $H$. midae consuming a prolineenriched abalone diet. Consecutive work on juvenile abalone will be worthwhile to determine if slow growth is noticeable at an earlier life stage and to establish if a proline-enriched diet can bridge the gap between the slow and fast-growing animals prior to adulthood.

\section{ACKNOWLEDGMENTS}

We acknowledge the North-West University (NWU) and the Technology Innovation Agency (TIA) of the Department of Science and Technology of South Africa for financial incentives. An additional word of thanks to HIK Abalone Farm (Pty) Ltd for hosting our feeding trial and for donating abalone for research purposes.

\section{ORCID}

Jeremie Zander Lindeque (iD https://orcid.org/0000-0001-8017-4278

\section{REFERENCES}

Angell, A. R., Pirozzi, I., De Nys, R., \& Paul, N. A. (2012). Feeding preferences and the nutritional value of tropical algae for the abalone Haliotis asinina. PLoS ONE, 7, e38857.

Bae, J. H., Yoon, S. H., \& Lim, S. Y. (2011). A comparative of heavy metal contents and biochemical characteristics of Japanese (Haliotis discus) and Korean abalone (Haliotis discus hannai). Food Science and Biotechnology, 20, 273-276.

Ballantyne, J. S., \& Moyes, C. D. (1987). The role of divalent cations and ionic strength in the osmotic sensitivity of glutamate oxidation in oyster gill mitochondria. Journal of Experimental Biology, 130, 203-217.

Brosnan, J. T. (2000). Glutamate, at the interface between amino acid and carbohydrate metabolism. The Journal of Nutrition, 130, 988S-990S.

Dettmer, K., Aronov, P. A., \& Hammock, B. D. (2007). Mass spectrometry-based metabolomics. Mass Spectrometry Reviews, 26, 51-78.

Difford, G., Vlok, A., Rhode, C., \& Brink, D. (2017). Heritability of growth traits in South African Abalone (Haliotis midae L.) using the 'internal reference' method. Aquaculture, 468, 451-457.

Dunn, W. B., Wilson, I. D., Nicholls, A. W., \& Broadhurst, D. (2012). The importance of experimental design and QC samples in largescale and MS-driven untargeted metabolomic studies of humans. Bioanalysis, 4, 2249-2264.

Ellis, S., \& Steyn, H. (2003). Practical significance (effect sizes) versus or in combination with statistical significance ( $p$-values): Research note. Management Dynamics. Journal of the Southern African Institute for Management Scientists, 12, 51-53.

Fleming, A. E., Van Barneveld, R. J., \& Hone, P. W. (1996). The development of artificial diets for abalone: A review and future directions. Aquaculture, 140, 5-53.

Garrett, R., \& Grisham, C. (2010). Biochemistry. Belmont, CA: Brooks Cole, Cengage Learning.

George, M. P., Presser, D., Szczesniewski, A., Yu, C., Mccain, K., \& Zhang, J. (2010). Simultaneous analysis of amino acids and acylcarnitines in dried blood spots. Application Note 5990-6036EN [Online], Application Note, 5990-6036EN.

Green, A. J., Jones, C. L., \& Britz, P. J. (2011). The protein and energy requirements of farmed South African abalone Haliotis midae L. cultured at optimal and elevated water temperatures. Aquaculture Research, 42, 1653-1663.

Halestrap, A. P. (1987). The regulation of the oxidation of fatty acids and other substrates in rat heart mitochondria by changes in the matrix volume induced by osmotic strength, valinomycin and $\mathrm{Ca} 2+$. Biochemical Journal, 244, 159-164.

Jarak, I., Tavares, L., Palma, M., Rito, J., Carvalho, R. A., \& Viegas, I. (2018). Response to dietary carbohydrates in European seabass (Dicentrarchus labrax) muscle tissue as revealed by NMR-based metabolomics. Metabolomics, 14, 95.

Krishnan, N., Dickman, M. B., \& Becker, D. F. (2008). Proline modulates the intracellular redox environment and protects mammalian cells against oxidative stress. Free Radical Biology and Medicine, 44, 671-681.

Laas, A., \& Vosloo, A. (2010). Exploring basic biochemical constituents in the body tissues of South African abalone Haliotis midae reared in shore-based mariculture systems. African Journal of Marine Science, 32, 55-63.

Li, P., Mai, K., Trushenski, J., \& Wu, G. (2009). New developments in fish amino acid nutrition: Towards functional and environmentally oriented aquafeeds. Amino Acids, 37, 43-53. https://doi.org/10.1007/ s00726-008-0171-1

Lindeque, J. Z., Hidalgo, J., Louw, R., \& Van Der Westhuizen, F. H. (2013). Systemic and organ specific metabolic variation in metallothionein knockout mice challenged with swimming exercise. Metabolomics, 9, 418-432.

Lindeque, J. Z., Matthyser, A., Mason, S., Louw, R., \& Taute, C. J. F. (2018). Metabolomics reveals the depletion of intracellular metabolites in HepG2 cells after treatment with gold nanoparticles. Nanotoxicology, 1-12.

Lindeque, J. Z., Jansen Van Rensburg, P. J., Louw, R., Van Der Westhuizen, F. H., Florit, S., Ramírez, L., ... Hidalgo, J. (2015). Obesity and metabolomics: Metallothioneins protect against high-fat diet-induced consequences in metallothionein knockout mice. Omics: A Journal of Integrative Biology, 19, 92-103. 
Lu, J., Feng, J., Cai, S., \& Chen, Z. (2017). Metabolomic responses of Haliotis diversicolor to organotin compounds. Chemosphere, 168, 860-869.

Macey, B., \& Coyne, V. (2005). Improved growth rate and disease resistance in farmed Haliotis midae through probiotic treatment. Aquaculture, 245, 249-261.

Mgaya, Y. D., \& Mercer, J. P. (1994). A review of the biology, ecology, fisheries and mariculture of the European abalone Haliotis tuberculata Linnaeus 1758 (Gastropoda: Haliotidae). Biology and Environment: Proceedings of the Royal Irish Academy, 285-304.

Newsholme, P., Bender, K., Kiely, A., \& Brennan, L. (2007). Amino acid metabolism, insulin secretion and diabetes. Biochemical Society Transactions, 35, 1180-1186.

Newsholme, P., Lima, M., Procopio, J., Pithon-Curi, T., Bazotte, R., \& Curi, R. (2003). Glutamine and glutamate as vital metabolites. Brazilian Journal of Medical and Biological Research, 36, 153-163.

Pérez-Arellano, I., Carmona-Álvarez, F., Martínez, A. I., Rodríguez-Díaz, J., \& Cervera, J. (2010). Pyrroline-5-carboxylate synthase and proline biosynthesis: From osmotolerance to rare metabolic disease. Protein Science, 19, 372-382.

Salway, J. G. (2004). Metabolism at a glance. Nashwille, TN: John Wiley $\&$ Sons.

Sheedy, J. R., Lachambre, S., Gardner, D. K., \& Day, R. W. (2015). 1H-NMR metabolite profiling of abalone digestive gland in response to shortterm starvation. Aquaculture International, 1-19.

Shinde, S., Villamor, J. G., Lin, W.-D., Sharma, S., \& Verslues, P. E. (2016). Proline coordination with fatty acid synthesis and redox metabolism of chloroplast and mitochondria. Plant Physiology, 172, 1074-1088.

Teulier, L., Weber, J.-M., Crevier, J., \& Darveau, C.-A. (2016). Proline as a fuel for insect flight: enhancing carbohydrate oxidation in hymenopterans. Proceedings of the Royal Society of London. Series B, Biological Sciences, 283, 20160333.

Troell, M., Robertson-Andersson, D., Anderson, R. J., Bolton, J. J., Maneveldt, G., Halling, C., \& Probyn, T. (2006). Abalone farming in South Africa: An overview with perspectives on kelp resources, abalone feed, potential for on-farm seaweed production and socioeconomic importance. Aquaculture, 257, 266-281. https://doi. org/10.1016/j.aquaculture.2006.02.066

Tung, C. H., \& Alfaro, A. C. (2012). Alternative protein sources in artificial diets for New Zealand's black-footed abalone, Haliotis iris, Martyn 1784, juveniles. Journal of the World Aquaculture Society, 43, 1-29.

Venter, L., Jansen Van Rensburg, P., Loots, D. T., Vosloo, A., \& Lindeque, J. Z. (2016). Untargeted metabolite profiling of abalone using gas chromatography mass spectrometry. Food Analytical Methods, 9, 1254-1261.

Venter, L., Jansen Van Rensburg, P. J., Loots, D. T., Vosloo, A., \& Lindeque, J. Z. (2017). From untargeted LC-QTOF analysis to characterisation of opines in abalone adductor muscle: Theory meets practice. Journal of Chromatography B, 1071, 44-48.

Venter, L., Lindeque, Z., Jansen Van Rensburg, P., Van Der Westhuizen, F., Smuts, I., \& Louw, R. (2015). Untargeted urine metabolomics reveals a biosignature for muscle respiratory chain deficiencies. Metabolomics, 11, 111-121.

Venter, L., Loots, D. T., Mienie, L. J., Van Rensburg, P. J. J., Mason, S., Vosloo, A., \& Lindeque, J. Z. (2018a). The cross-tissue metabolic response of abalone (Haliotis midae) to functional hypoxia. Biology Open, 7, bio031070.

Venter, L., Loots, D. T., Mienie, L. J., Van Rensburg, P. J. J., Mason, S., Vosloo, A., \& Lindeque, J. Z. (2018b). Uncovering the metabolic response of abalone (Haliotis midae) to environmental hypoxia through metabolomics. Metabolomics, 14, 49.
Venter, L., Loots, D. T., Vosloo, A., Jansen Van Rensburg, P., \& Lindeque, J. Z. (2018). Abalone growth and associated aspects: Now from a metabolic perspective. Reviews in Aquaculture, 10, 451-473.

Venter, L., Vosloo, A., Loots, D. T., Mienie, L. J., Van Rensburg, P. J. J., \& Lindeque, J. Z. (2018). Characterising the metabolic differences related to growth variation in farmed Haliotis midae. Aquaculture, 493, 144-152.

Viana, M. T., D'abramo, L. R., Gonzalez, M. A., García-Suárez, J. V., Shimada, A. \& Vásquez-Peláez, C. (2007). Energy and nutrient utilization of juvenile green abalone (Haliotis fulgens) during starvation. Aquaculture, 264, 323-329.

Vlok, A. C., Difford, G. F., Rhode, C., \& Brink, D. (2016). An assessment of hatchery cohort growth rates of South African abalone, Haliotis midae, across four commercial environments. Journal of the World Aquaculture Society, 47, 658-666.

Vosloo, D., Van Rensburg, L., \& Vosloo, A. (2013). Oxidative stress in abalone: The role of temperature, oxygen and L-proline supplementation. Aquaculture, 416, 265-271. https://doi.org/10.1016/j. aquaculture.2013.09.031

Wehrens, R., Hageman, J. A., Van Eeuwijk, F., Kooke, R., Flood, P. J., Wijnker, E., ... Hall, R. D. (2016). Improved batch correction in untargeted MS-based metabolomics. Metabolomics, 12, 1-12.

Wu, G., Bazer, F. W., Dai, Z., Li, D., Wang, J., \& Wu, Z. (2014). Amino acid nutrition in animals: Protein synthesis and beyond. Annual Review of Animal Biosciences, 2, 387-417.

Wu, G., Bazer, F. W., Burghardt, R. C., Johnson, G. A., Kim, S. W., Knabe, D. A., ... Satterfield, M. C. (2011). Proline and hydroxyproline metabolism: Implications for animal and human nutrition. Amino Acids, 40, 1053-1063.

Xia, J., Mandal, R., Sinelnikov, I. V., Broadhurst, D., \& Wishart, D. S. (2012). MetaboAnalyst 2.0-a comprehensive server for metabolomic data analysis. Nucleic Acids Research, 40, W127-W133.

Xia, J., Sinelnikov, I. V., Han, B., \& Wishart, D. S. (2015). MetaboAnalyst 3.0-making metabolomics more meaningful. Nucleic Acids Research, 43, 251-257.

Xu, J., Wang, F., Jakovlić, I., Prisingkorn, W., Li, J.-T., Wang, W.-M., \& Zhao, Y.-H. (2018). Metabolite and gene expression profiles suggest a putative mechanism through which high dietary carbohydrates reduce the content of hepatic betaine in Megalobrama amblycephala. Metabolomics, 14, 94. https://doi.org/10.1007/ s11306-018-1389-x

Yang, C., Hao, R., Du, X., Deng, Y., Sun, R., \& Wang, Q. (2018). Metabolomics responses of pearl oysters (Pinctada fucata martensii) fed a formulated diet indoors and cultured with natural diet outdoors. Frontiers in Physiology, 9.

\section{SUPPORTING INFORMATION}

Additional supporting information may be found online in the Supporting Information section at the end of the article.

How to cite this article: Venter L, Mienie LJ, Vosloo A, Loots DT, Jansen van Rensburg P, Lindeque JZ. Effect of prolineenriched abalone feed on selected metabolite levels of slow-growing adult Haliotis midae. Aquac Res. 2019;00:1-11. https://doi.org/10.1111/are.13978 